\title{
Planning Functional Grasps of Simple Tools Invokes the Hand-independent Praxis Representation Network: An fMRI Study
}

INS is approved by the American Psychological Association to sponso Continuing Education for psychologists. INS maintains responsibility for this program and its content.

\author{
Łukasz Przybylski, AND Gregory Króliczak \\ Action \& Cognition Laboratory, Institute of Psychology, Adam Mickiewicz University in Poznan, Poland
}

(Received May 14, 2016; Final Revision November 5, 2016; AccePTed November 15, 2016)

\begin{abstract}
Objectives: Neuropsychological and neuroimaging evidence indicates that tool use knowledge and abilities are represented in the praxis representation network (PRN) of the left cerebral hemisphere. We investigated whether PRN would also underlie the planning of function-appropriate grasps of tools, even though such an assumption is inconsistent with some neuropsychological evidence for independent representations of tool grasping and skilled tool use. Methods: Twenty right-handed participants were tested in an event-related functional magnetic resonance imaging (fMRI) study wherein they planned functionally appropriate grasps of tools versus grasps of non-tools matched for size and/or complexity, and later executed the pantomimed grasps of these objects. The dominant right, and non-dominant left hands were used in two different sessions counterbalanced across participants. The tool and non-tool stimuli were presented at three different orientations, some requiring uncomfortable hand rotations for effective grips, with the difficulty matched for both hands. Results: Planning functional grasps of tools ( $v s$. non-tools) was associated with significant asymmetrical increases of activity in the temporo/occipital-parieto-frontal networks. The greater involvement of the left hemisphere PRN was particularly evident when hand movement kinematics (including wrist rotations) for grasping tools and non-tools were matched. The networks engaged in the task for the dominant and non-dominant hand were virtually identical. The differences in neural activity for the two object categories disappeared during grasp execution. Conclusions: The greater hand-independent engagement of the left-hemisphere praxis representation network for planning functional grasps reveals a genuine effect of an early affordance/function-based visual processing of tools. (JINS, 2017, 23, 108-120)
\end{abstract}

Keywords: Affordances, Action planning, Motor cognition, Pantomimed execution, Tool use, Functional neuroimaging

\section{INTRODUCTION}

Tools, utensils, and other implements (hereafter "tools") play a critical role in our daily functioning. Therefore, many neuropsychological and neuroimaging studies have investigated how tool knowledge and tool use abilities are represented in the human brain (Frey, 2007; Haaland \& Harrington, 1996; Vingerhoets, 2014). Among the necessary skills essential for an effective use of tool functions is the reconciliation of the intended action goals with knowledge on physical aspects of the to-be-used objects. Namely, graspable parts that enable their proper handling and use must be identified first.

Meanwhile, most projects using functional magnetic resonance imaging (fMRI) have focused on the neural bases

Correspondence and reprint requests to: Grzegorz Króliczak, Instytut Psychologii UAM, Ul. Szamarzewskiego 89, 60-568 Poznań, Poland. E-mail: krolgreg@amu.edu.pl of planning pantomimed actions with tools (Johnson-Frey, Newman-Norlund, \& Grafton, 2005; Kroliczak \& Frey, 2009; Maki-Marttunen, Villarreal, \& Leiguarda, 2014; with linguistic cues, and/or participants imagining tools in easyto-use orientations) or execution of such pantomimes and/or real tool use (Brandi, Wohlschlager, Sorg, \& Hermsdorfer, 2014; Hermsdorfer, Terlinden, Muhlau, Goldenberg, \& Wohlschlager, 2007; Valyear, Gallivan, McLean, \& Culham, 2012; Vingerhoets, Vandekerckhove, Honore, Vandemaele, \& Achten, 2011). Alternatively, the task was identification of implements that are best for realization of action goals (Mizelle, Kelly, \& Wheaton, 2013), or matching of hand postures to object usage (Vingerhoets, Nys, Honore, Vandekerckhove, \& Vandemaele, 2013; cf. Buxbaum, Kyle, Tang, \& Detre, 2006).

Remarkably, such a fundamental skill as the ability to properly grasp tools, involving neural computations that must precede the ones for their effective use is conspicuously 
missing in this debate (see also Fabbri, Stubbs, Cusack, \& Culham, 2016). In short, previous neuroimaging reports on tool-use pantomimes omitted the grasp component, and studies on real tool use did not assess its contribution to the task-related activity. Occasionally, function-appropriate grasps were studied in isolation, in the context of imagined or pantomimed tool grips/grip postures (Creem-Regehr \& Lee, 2005; Vingerhoets \& Clauwaert, 2015), without considering a progression from the planning through execution of functional grasps.

Yet, preparatory phases including the retrieval of knowledge on functional objects, and how to handle them, must be completed before a tool or utensil is grasped and any action performed (Garofeanu, Kroliczak, Goodale, \& Humphrey, 2004; Goodale, Kroliczak, \& Westwood, 2005). Indeed, the lack of neuroimaging studies on planning functional grasps of tools is quite unexpected given neuropsychological evidence that representations that underlie functional grasping versus using tools can be dissociated (Randerath, Goldenberg, Spijkers, Li, \& Hermsdorfer, 2010).

Here, we investigate the neural underpinnings of the planning and execution of functional grasps directed at tools compared to control grasps of objects with similar sizes and structures but no obvious functions. To this end, fMRI was used to study blood-oxygen-level-dependent (BOLD) signal modulations while participants watched tools with an intention to plan and perform pantomimed functional grasps (appropriate for an immediate use) versus spontaneous grasps of non-tools. The neural activity was examined both for the dominant and non-dominant hand. Based on earlier studies (Kroliczak \& Frey, 2009; Kroliczak, McAdam, Quinlan, \& Culham, 2008), we hypothesized that the planning of function-appropriate grasps would involve the praxis representation network (PRN; Frey, 2008).

PRN is a left hemisphere network of areas whose task is to integrate and transform conceptual and sensorimotor information into purposeful, skilled acts (praxis), including the processing/retrieval and control of tool-use pantomimes and other symbolic gestures (Frey, 2007; Kroliczak \& Frey, 2009; Kubiak \& Kroliczak, 2016). Its major temporo-parietofrontal nodes are shown in Figure 1. There is convincing evidence that the anterior supramarginal gyrus (aSMG) and anterior intraparietal sulcus (aIPS) plays a critical role in the processing, planning, and performance of skilled manual actions (Goldenberg \& Spatt, 2009; Kroliczak, Piper, \& Frey, 2016; Peeters, Rizzolatti, \& Orban, 2013).

Yet, an interplay with the caudal middle temporal gyrus (cMTG) and rostral middle frontal gyrus (rMFG) is necessary for the retrieval of core visual features of tools, their concepts and the knowledge on possible or typical actions afforded by them (Goldenberg, Hartmann, \& Schlott, 2003; Haaland, Harrington, \& Knight, 2000; Kristensen, Garcea, Mahon, \& Almeida, 2016; Watson \& Buxbaum, 2015), the selection of action goals, and the appropriate object-response associations (Buxbaum, Shapiro, \& Coslett, 2014; Goldenberg \& Spatt, 2009; Vannuscorps, Dricot, \& Pillon, 2016; see also Ishibashi, Pobric, Saito, \& Lambon

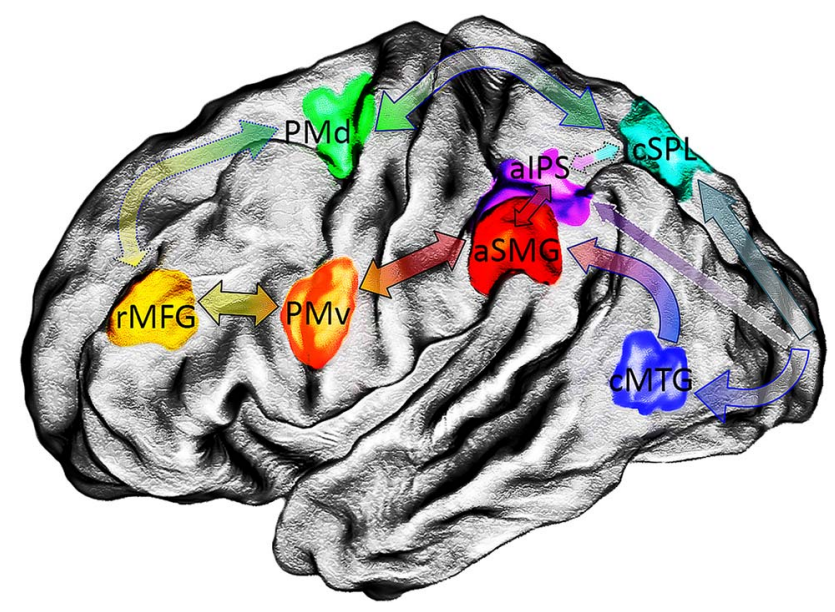

Fig. 1. A schematic of the praxis representation network (PRN) and the putative connections between its areas. PRN is associated with the left cerebral hemisphere wherein the integration and transformation of perceptual, conceptual, and sensorimotor information takes place for the control of skilled manual actions. The whole network consists of the caudal middle temporal gyrus (cMTG), anterior supramarginal gyrus (aSMG), anterior intraparietal sulcus (aIPS), caudal superior parietal lobule (cSPL), ventral and dorsal premotor cortices (PMv and PMd, respectively), and rostral middle frontal gyrus (rMFG).

Ralph, 2016). Thus, PRN plays a critical role in goal-directed motor cognition.

To maximize a chance of activating the critical components of PRN, only simple tools were used, namely objects whose primary function is an extension or reinforcement of the hands during daily actions, and the tasks are well learned (Frey, 2008). We expected that PRN would be more engaged for planning tool grips even when the graspable parts of tools and non-tools and the related grasp kinematics are closely matched. The greater involvement of PRN would then point to the importance of function-oriented processing (Gibson, 1977; Mizelle et al., 2013). Finally, the left-lateralized activity associated with planning functional grasps was expected to be similar, independent of the hand used for task performance (Kroliczak \& Frey, 2009; Vingerhoets et al., 2012).

\section{METHODS}

\section{Participants}

Twenty native Polish speaking volunteers (10 females), mainly students of Adam Mickiewicz University in Poznan, ranging in age from 19 to 24 years (mean age $=22.7$ years; $S D=1.6)$ participated in two counterbalanced experiments testing their dominant right and non-dominant left hands, respectively. All participants were strongly right-handed (Edinburgh Handedness Inventory index: $M=92.9$; $S D=13.9$; Oldfield, 1971), had normal or correctedto-normal visual acuity, and none had a history of 
neurological/psychiatric disorders. All participants completed a pre-scan MRI safety questionnaire, were familiarized with the study protocols, and gave their written informed consents. At study conclusion they were reimbursed financially for their time and efforts, and debriefed. The Bio-Ethics Committee at Poznan University of Medical Sciences approved the protocols and procedures, which conformed to the principles of the 2013 WMA Helsinki Declaration.

\section{The Stimuli and Set-up}

Twelve familiar tools were photographed and used as stimuli. Some of these objects, for example a rake or screwdriver, serve as an arm extension, others, namely a hammer or knife, as a reinforcement of the hand. Their most common/typical functions were studied and participants were extensively instructed/trained. Twelve control stimuli with no obvious functions were either natural fragments of wooden sticks or man-made objects. An attempt was made to match graspable parts of these items, their general visual appearance or structural complexity, that is number of parts, and their size/ surface area. The examples of stimuli in 3 different orientations are shown in Figure 2A, and all the remaining objects are presented in panel $\mathrm{B}$ and $\mathrm{C}$.

Each object was photographed using a Sony Cybershot DSC H2 digital-reflex camera on a white background, in three different orientations: 0, 135, 225 degrees (as in Garofeanu et al., 2004). The $0^{\circ}$ orientation, with a tool handle pointing toward 12:00 or 0:00 o'clock, typically demanded an uncomfortable hand rotation for the functional grasp regardless of the hand used. The $135^{\circ}$ orientation, with a tool handle pointing between 4 and 5 , and the $225^{\circ}$ orientation, with a handle pointing between 7 and 8 , were less demanding. The former required easier hand rotations for the right, and the latter for the left hand, and as complementary they were also matched for both hands. For non-tools, the objects' $0^{\circ}$ orientation would be associated with the most convenient grasps. Therefore, the $135^{\circ}$ and $225^{\circ}$ orientations were critical: only in these two conditions hand movement kinematics such as wrist rotations, and grip apertures were matched for tools and non-tools.

All objects were shown in foreshortened perspectives as if viewed by a person standing by the table on which they were put. The images were displayed one at a time, in grayscale, using NordicNeuroLab 40" 4K UHD InroomViewingDevice (http://www.nordicneurolab.com) positioned $60 \mathrm{~cm}$ behind the scanner bore and viewed via a mirror attached to the head coil. SuperLab 4.5.4 (http://www.superlab.com) installed on a MacBook Pro computer (with OSX v10.8) was used for stimulus presentation. The beginnings of trials, but not the timing of events within trials, were controlled by the MRI scanner triggers. During grasp planning, participants' fingers rested on the LU400-Pair response pads (http://cedrus.com/ lumina/) positioned near the legs. Hence, grasp movement onsets were recorded. These movements were also constantly monitored by the experimenter to immediately detect
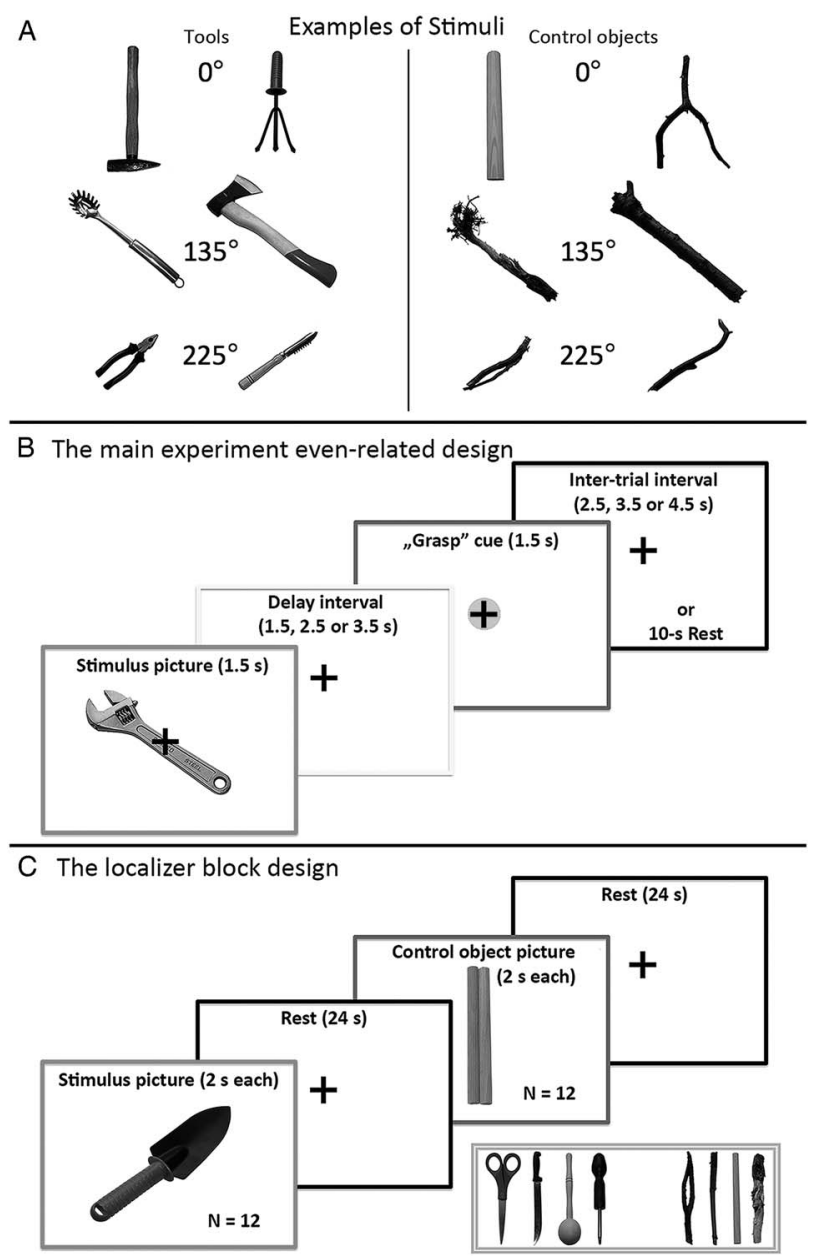

Fig. 2. Stimuli and designs. A: Examples of stimuli used in our study. (Left panel) Tools, and (right panel) control objects, presented at 3 different orientations. B: Trial structure and timing of the main study with an event-related design. The stimulus picture was followed by a variable delay interval for grasp planning, and a "Go" cue for the pantomimed execution of the pre-planned grasp. Trials concluded with pseudo-randomly introduced variable inter-trial intervals (ITIs) or rest intervals. C: Trial structure and timing of the localizer scans with a block design. There were 5 blocks in which participants simulated the use of the depicted tools, five blocks of non-tool objects in which participants manually counted the number of object parts, and five blocks of rest periods, all presented in pseudo-random order. The inset at the bottom shows all the remaining objects.

incorrect responses, such as movement onsets during planning or wrong hand rotations during grasp performance. Participants' heads were supported and immobilized with padding. Thus, hand movements during task execution did not easily translate into head displacements.

\section{Procedure}

An event-related paradigm was used in the main study. Participants completed two separate sessions on consecutive days, using their dominant right and the non-dominant left hands, counterbalanced. Each functional run comprised of 
24 trials, 12 involving tools and 12 non-tools, 6 items of each type per single run, 6 presented at the $0^{\circ}$ orientation, and 6 at $135^{\circ}$ and 225 . Four runs were required to use each of the 12 objects in $135^{\circ}$ and $225^{\circ}$ orientations. Therefore, each participant was initially tested with 4 pseudorandom orders of tools and non-tools assigned to the first 4 runs. An additional $5^{\text {th }}$ run comprised pseudorandom combination of all stimuli, counterbalanced across the two sessions.

Thus, each participant received a different sequence of target objects. All stimuli were presented in the same central location marked with a black cross which reminded participants to pay attention to the presented objects. There was no specific instruction about how the grasp planning should be performed. It was emphasized that hand movements and their rotations should be as precise as possible, that an upper arm should remain as static as possible, and head motion must be avoided.

Each trial consisted of an initial variable ISI of $0,0.25,0.5$, or $0.75 \mathrm{~s}$, which ensured that stimulus onset occurred at variable delays relative to the onset of the acquisition of functional volumes, followed by a 1.5 -s visual presentation of a stimulus object and a variable 1.5, 2.5, or 3.5-s delay interval for grasp planning. Subsequently, the "GRASP" cue, a central green circle lasting $1.5 \mathrm{~s}$, was shown to prompt participants to simulate preplanned grasps. A trial concluded with an obligatory variable inter-trial interval (ITI) of 2.5, 3.5, or $4.5 \mathrm{~s}$ plus time required for synchronization with the scanner trigger. Notably, each object type was followed equally often by each of the 3 delay intervals. Similarly, each grasp type was also followed equally often by each of the 3 ITIs. In each run there were also six additional 5.5-s periods introduced pseudorandomly between trials with the longest ITIs, which resulted in six 10-s time intervals serving as resting baseline. Trial structure and timing is shown in Figure 2B. The timing of events within trials is consistent with an oversampling method (Miezin, Maccotta, Ollinger, Petersen, \& Buckner, 2000) suitable for rapid, event-related fMRI testing.

\section{Additional Localizer Scans}

All participants were tested in a functional Tool Use Localizer (TUL) whose goal was to independently reveal the areas belonging to the PRN. Importantly, our TUL involved simulated grips and subsequent tool use pantomimes. The control task was "manual counting" of non-tool parts, wherein an inversion of a palm with an extended index finger meant one part, the index finger and thumb meant two parts, and an inversion of a palm and the extension of a thumb, index, and middle finger meant three or more parts. Notably, native speakers of Polish do not count this way; typically, the hand is positioned as in a hitch-hiking gesture and counting starts with a thumb. A single stick or shaft was treated as having one part, few objects had two distinct parts, while some objects were more complex and by definition would have three or more parts.

There were five 24-s blocks of a pantomimed "graspand-tool-use" task in response to 12 tool stimuli displayed for $2 \mathrm{~s}$, and five 24-s blocks of counting parts in response to 12 non-tool stimuli shown for $2 \mathrm{~s}$. Additional five 24-s blocks of rest periods were introduced pseudorandomly between task blocks. Time structure and timing is illustrated in Figure $2 \mathrm{C}$. The stimuli and their number was exactly the same as in the main experiment. Participants were to disregard changes in orientation during TUL. There were two different pseudorandom orders of task and rest blocks assigned pseudorandomly across hands used on a given day and testing sessions.

\section{Data Acquisition}

Scanning was performed on a Siemens (Erlangen, Germany) 3 Tesla Trio MRI scanner in the Laboratory of Brain Imaging (http://lobi.nencki.gov.pl) at the Nencki Institute of Experimental Biology (http://en.nencki.gov.pl) in Warsaw, Poland. The MRI scanner was equipped with echo planar imaging (EPI) capabilities and a 32-channel PA head coil for radio frequency transmission and signal reception. Before the beginning of functional runs, Auto Align Scout and True FISP sequences were used to help with slice prescription. The blood-oxygen-level-dependent (BOLD) echoplanar images were collected using a $\mathrm{T}_{2}^{*}$-weighted gradient echo sequence: time repetition $(\mathrm{TR})=2000 \mathrm{~ms}$; time echo $(\mathrm{TE})=30 \mathrm{~ms}$; flip angle $=90^{\circ} ; 64 \times 64$ matrix; field of view $(\mathrm{FOV})=$ $200 \mathrm{~mm}$; 35 contiguous axial slices, 3.1-mm isotropic voxels.

There were 145 volumes acquired in each run of the main experiment, and 194 volumes in each of the localizer scans. High-resolution $\mathrm{T}_{1}$-weighted structural images were acquired using magnetization prepared rapid gradient echo (MP-RAGE) pulse sequence: $\mathrm{TR}=2530 \mathrm{~ms}$; $\mathrm{TE}=3.32 \mathrm{~ms}$; inversion time $(\mathrm{TI})=1200 \mathrm{~ms} ; \mathrm{FA}=7^{\circ} ; 256 \times 256$ voxel matrix size; FOV $=256 \mathrm{~mm} ; 176$ contiguous axial slices; $1.0-\mathrm{mm}$ isotropic voxels. To enhance registration between functional EPIs and structural T1-weighted images, fast spin echo T2-weighted structural images were also acquired with the following parameters: $\mathrm{TR}=3200 \mathrm{~ms}$; $\mathrm{TE}=402 \mathrm{~ms}$; $\mathrm{FA}=120^{\circ} ; 512 \times 512$ voxel matrix size; $\mathrm{FOV}=256 \mathrm{~mm}$; 176 contiguous sagittal slices; $0.5-0.5-1 \mathrm{~mm}$ non-isotropic voxels. Raw data were converted to NIFTI-1 format with MRI-Convert software (http://lcni.uoregon.edu/downloads/ mriconvert).

\section{Image Analyses}

Structural T1- and T2-weighted images were processed using FSL (FMRIB's Software Library, http://fsl.fmrib.ox.ac. uk/fsl/fslwiki/) v5.0.6 (Jenkinson, Beckmann, Behrens, Woolrich, \& Smith, 2012) and FreeSufer v5.3 (Fischl, 2012). MP-RAGE scans were first averaged using FMRIB Linear Image Registration Tool because for all participants two such images were obtained. Further pre-processing involved a pipeline described by Bidula and Kroliczak (2015). Functional images were analyzed with FSL's FMRI Expert Analysis Tool v.6.00 (Jenkinson et al., 2012). The non-brain tissues were removed using the FSL's Brain Extraction Tool, 
and head motion was corrected with the FSL motion correction algorithm. The average absolute head displacement equaled $0.162 \mathrm{~mm}$ (the range was $0.04-0.61 \mathrm{~mm}$ ) when participants used the right hands, and $0.176 \mathrm{~mm}(0.06-0.43 \mathrm{~mm})$ when the left hands were used. The images were spatially smoothed with a Gaussian kernel of full-width-halfmaximum $=6.2 \mathrm{~mm}$ (twice the voxel size), and temporally smoothed using high-pass filtering $\sigma=50.0 \mathrm{~s}$.

Each fMRI run was analyzed separately at the first level. Before statistical analyses, autocorrelation in the data was corrected using a pre-whitening procedure (Woolrich, Ripley, Brady, \& Smith, 2001). Hemodynamic responses were modeled using a double-gamma function. In the main experiment, planning-related activity was modeled as the 3.0-s period beginning with the onset of the stimulus picture and lasting through the end of the shortest 1.5-s delay interval (see Figure 2B). Execution-related activity was modeled as the 1.5-s period during which the "grasp" cue was visible.

In the localizer experiment, activity from the whole 24-s blocks was modeled. The runs from a given experiment were averaged using the fixed effects model. The group analyses were performed using random-effects components of mixedeffects variance modeled and estimated with FLAME Stage 1 procedure (Beckmann, Jenkinson, \& Smith, 2003). The resulting $\mathrm{Z}$ (Gaussianized $\mathrm{t} / \mathrm{F}$ ) statistic images were thresholded using the FSL's settings of $\mathrm{Z}>3.1, p=.001$, and corrected for multiple comparisons using clusterwise significance threshold of $p=.05$. Namely, family-wise error rate (FWER) was controlled at $\alpha=0.05$ (Eklund, Nichols, \& Knutsson, 2016).

Hand-independent activity was obtained using inclusive contrast masking (Nichols, Brett, Andersson, Wager, \& Poline, 2005). To directly compare neural activities across hands and tasks, 2 (Hand: right, left) $\times 2$ (Task: grasp planning, grasp execution or pantomimed tool use) repeated-measures analyses of variance (ANOVAs) were run. Anatomical locations of clusters with significant activity were established with help from the Juelich Histological, and Harvard-Oxford probabilistic atlases implemented in the FSL. These clusters were visualized using the CARET software mapping onto the human Population-Average, Landmark- and Surface-based (PALS) brain atlas (Van Essen, 2005).

\section{Region of Interest Analyses}

Six regions of interest (ROIs) for the right, and six for the left hand were created: cMTG, aSMG, rMFG, the caudal superior parietal lobule (cSPL), ventral premotor cortex (PMv), and dorsal premotor cortex (PMd). They were 5-mm radius spheres centered on peak voxels from clusters involved in planning functional versus control grasps. Using FSL FEATQuery, mean percent signal change values within these ROIs were calculated from all voxels for planning functional or control grasps versus resting baseline. Subsequently 2 (Hand: right, left) $\times 2$ (Task: planning, execution) $\times 2$ (Object: tool, control) repeated-measures ANOVAs were run separately for each ROI from the two sets, with post hoc Bonferroni correction for multiple comparisons.

\section{RESULTS}

\section{Planning Functional Grasps of Tools versus Control Objects with the Right Hand}

Significant clusters of activity were observed in a network of areas associated with PRN. The results are shown in Figure 3A. The cMTG/lateral occipital cortex (LOc), aSMG, and cSPL activity was exclusively left lateralized, aIPS showed left-hemisphere advantage, whereas PMv and PMd was engaged more symmetrically. There was also significant activity in the left antero-dorsal precuneus (adPreCun), bilaterally in supplementary/pre-supplementary motor area (SMA-complex), and right anterior insular cortex (aIC). As shown in Figure 3B, when the planned movement kinematics were accounted for by contrasting trials involving object orientations of 135 and $225^{\circ}$, the parieto-frontal activity was now almost exclusively left lateralized. The only exception was a small cluster in aIPS.

\section{Planning Grasps of Control Objects versus Grasps of Tools with the Right Hand}

Consistent with our earlier study (Marangon, Kubiak, \& Kroliczak, 2016), signal modulations in the angular gyri (AG), bilateral ventral PreCun, and mid-to-posterior middle and superior temporal gyri (mpMTG/STG) were revealed. The pattern, illustrated in Supplementary Figure 1A, results from greater inhibition of these areas for the more difficult task of planning grasps of tools.

\section{Execution of Functional Grasps of Tools versus Controls with the Right Hand}

No significant cluster of activity was detected. The inverse contrast was empty, too.

\section{Planning Functional Grasps of Tools versus Control Objects with the Left Hand}

As shown in Figure 3C, cMTG/LOc, aSMG, cSPL, and PMv activity was exclusively left lateralized, whereas aIPS, PMd, SMA-complex, adPreCun, and aIC were engaged bilaterally. There was additional involvement of left rMFG, and handdependent engagement of the right sensorimotor cortices (SI-MI). When movement kinematics were matched, greater PRN involvement was still observed. While cSPL and rMFG no longer played a role, there were additional contributions from right aIPS and SI-MI. This effect is shown in Figure 3D. Direct contrasts of activity associated with the right and left hand, as depicted in Supplementary Figure 1B, showed significant differences only within their respective SI-MIs. 

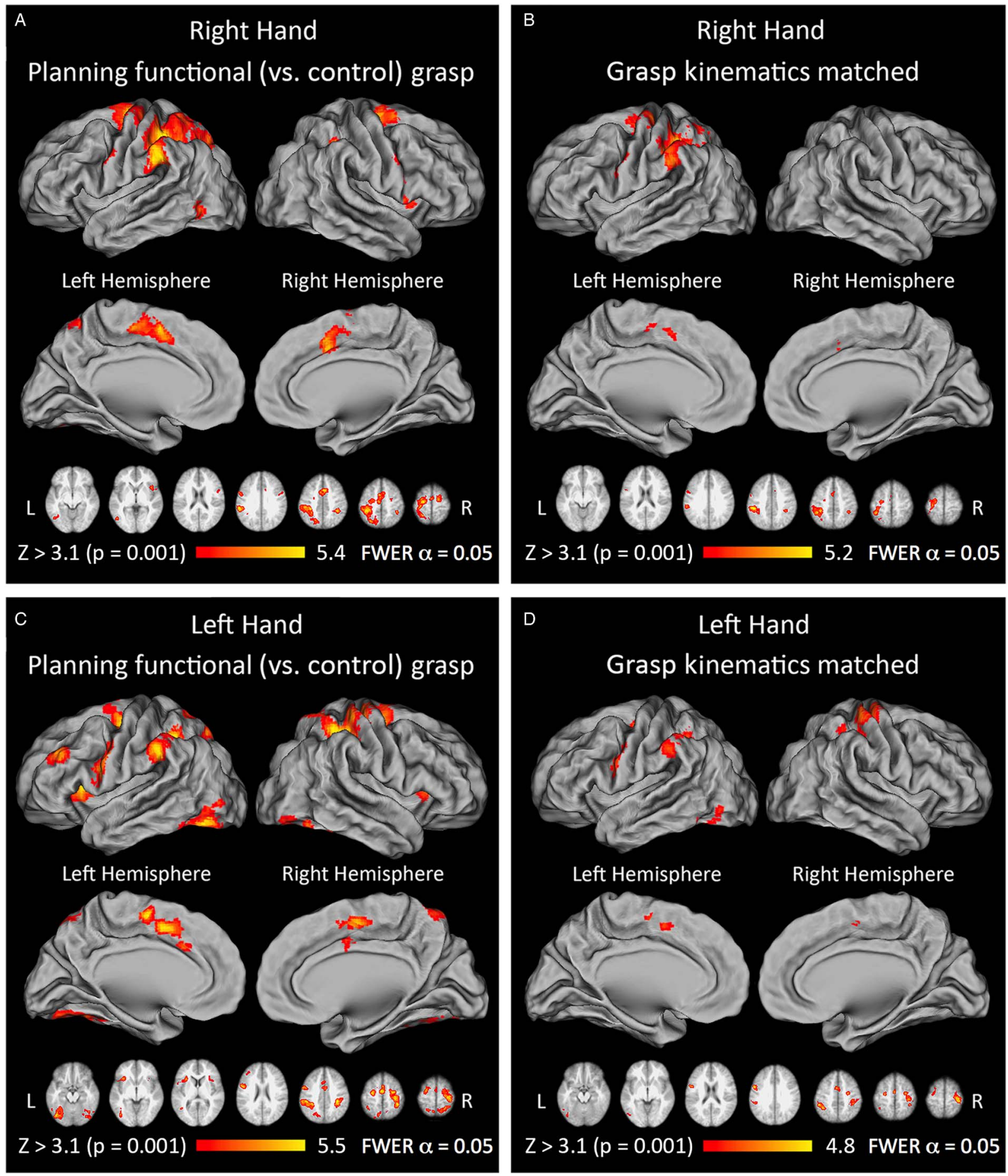

Fig. 3. Brain areas showing significantly greater neural activity during the planning of functional grasps with the dominant right (A,B), and non-dominant left (C,D) hand. Functional brain activity in representative slices and volumetric surface renderings are shown. A: Planning functional grasps of tools versus planning control grasps of non-tools with the right hand, regardless of object orientation. All the left-hemisphere areas belonging PRN were invoked, except for rMFG. The aIPS showed left-hemisphere advantage, while PMv and PMd was engaged bilaterally. The left antero-dorsal precuneus (adPreCun), bilateral supplementary/pre-suplementary motor area (SMAcomplex), and right anterior insular cortex (aIC) were also involved. B: Planning functional versus control grasps for the right hand with forearm/grip kinematics matched. Almost exclusively left-lateralized increases in PRN were observed, except for additional involvement of right aIPS. C: Planning functional grasps of tools versus control grasps of non-tools with the non-dominant left hand, regardless of object orientation. All the critical areas of PRN were involved, with aIPS and PMd in both hemispheres. Ventral visual areas, SMAcomplex, adPreCun, and aIC were engaged bilaterally. D: Planning functional versus control grasps for the non-dominant left hand with forearm/grip kinematics matched. As for the right hand, the involved network was almost entirely left lateralized, with additional contributions from the right aIPS and sensorimotor cortices. FWER = family-wise error rate. 


\section{Planning Grasps of Control Objects versus Grasps of Tools with the Left Hand}

Only right AG engagement was revealed and it is depicted in Supplementary Figure 1C.

\section{Execution of Functional Grasps of Tools versus Controls with the Left Hand}

No significant cluster of activity was detected. The inverse contrast was empty, too.

\section{Hand-Independent Networks for Planning Functional Grasps and Pantomimed Tool Use}

As shown in Figure 4A, all the major left-hemisphere areas invoked for functional grasp planning were largely overlapping with or even located within a greater network of regions involved in pantomimed tool use. Unexpectedly, rMFG did not make the threshold. There was also some bilateral activity in mid IPS, SMA, mid-to-caudal left SPL, right $\mathrm{PMd}, \mathrm{PMv}$, and aIC. Figure 4B shows a direct comparison of functional grasp planning and pantomimed tool use collapsed across hands. All the areas belonging to PRN and their right-hemisphere counterparts, depicted in dark reds, were more engaged in grasp planning. This was the case regardless of whether or not visual and motor demands were controlled.

Notably, in this panel the activity from control tasks was subtracted. The inverse contrast between pantomimed tool use and planning functional grasp revealed bilateral modulations in AG, vPreCun, mpMTG/STG, SI-MI, and

Fig. 4. Significant brain activity for planning functional grasp and pantomimed tool use, and direct comparisons of planning and execution of the studied tasks. A: Hand-independent activity for planning functional grasp and pantomimed tool use overlaid on each other. Nearly all the left-hemisphere areas invoked for functional grasp planning were located within a greater network of regions engaged for pantomimed tool use. Notably, mid-to-caudal left IPS and cSPL were devoted exclusively for grasp planning. B: Functional grasp planning versus pantomimed tool use. All the PRN areas and their right-hemisphere counterparts were significantly more involved in functional grasp planning (as shown in warm colors, primarily red). Some of the regions that showed significantly greater engagement for pantomimed tool use (shown in blue) belong putatively to the default mode network (DMN; Raichle et al., 2001), and were less inhibited during this task. This panel also shows that, when visual and motor demands were controlled, aSMG and aIPS (shown in orange) were significantly more involved in pantomimed tool use when compared to grasp execution. C: Functional grasp planning versus grasp execution. When visual and motor demands were controlled, almost all lefthemisphere areas of PRN were involved significantly more in grasp planning (shown in red). Additional, activity was found in the right early visual, parietal and sensorimotor regions. The inverse contrast (depicted in blue) showed greater grasp-execution related signal modulations outside of PRN. dorsal lateral/medial prefrontal cortices. In our earlier study (Marangon et al., 2016), such a network was revealed for an easier manual task. Figure 4B also demonstrates that pantomime tool use invokes left aSMG/aIPS, right SI and
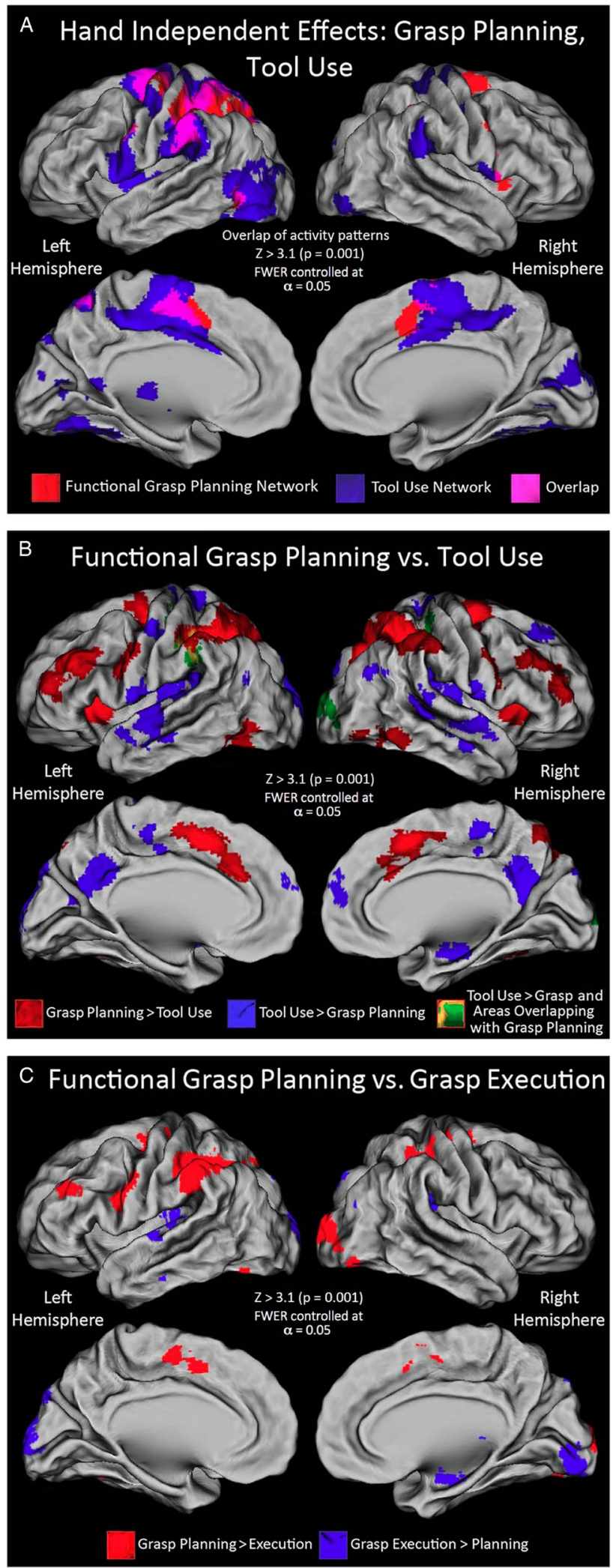


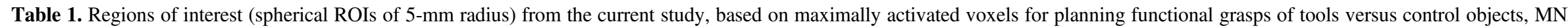
coordinates of their peak voxels, their $\mathrm{Z}$ values, and the results of statistical analyses

\begin{tabular}{|c|c|c|c|c|c|c|c|c|c|}
\hline \multirow[b]{2}{*}{ Left hemisphere region } & \multirow[b]{2}{*}{ Hand } & \multicolumn{3}{|c|}{ MNI coordinates } & \multirow{2}{*}{$\begin{array}{l}\text { Peak } Z \\
\text { value }\end{array}$} & \multirow{2}{*}{$\begin{array}{l}\text { Main effect } \\
\text { of hand } \\
\text { (right, left) }\end{array}$} & \multirow{2}{*}{$\begin{array}{c}\text { Main effect } \\
\text { of task } \\
\text { (plan, exec.) }\end{array}$} & \multirow{2}{*}{$\begin{array}{l}\text { Main effect } \\
\text { of object } \\
\text { (tool, contr.) }\end{array}$} & \multirow[b]{2}{*}{ Interactions } \\
\hline & & $\mathrm{x}$ & $\mathrm{y}$ & $\mathrm{z}$ & & & & & \\
\hline \multirow{2}{*}{$\begin{array}{l}\text { Caudal middle temporal gyrus } \\
\text { (cMTG) / lateral occipital (LO) } \\
\text { cortex }\end{array}$} & Right & -48 & -66 & -2 & 4.2 & \multirow[t]{2}{*}{0.26} & $*$ & $* * *$ & \multirow[t]{2}{*}{ ns. } \\
\hline & Left & -42 & -72 & -4 & 4.37 & & $\mathrm{PES}=0.27, \mathrm{OP}=0.71$ & $\mathrm{PES}=0.81, \mathrm{OP}=1.0$ & \\
\hline \multirow{2}{*}{$\begin{array}{l}\text { Anterior supramarginal gyrus } \\
\text { (aSMG) }\end{array}$} & Right & -54 & -34 & 38 & 5.42 & \multirow[t]{2}{*}{0.32} & \multirow[t]{2}{*}{0.18} & $* * *$ & $(\mathrm{~T} \times \mathrm{O}) * * *$ \\
\hline & Left & -52 & -34 & 36 & 5.15 & & & $\mathrm{PES}=0.88, \mathrm{OP}=1.0$ & $\mathrm{PES}=0.61, \mathrm{OP}=0.99$ \\
\hline \multirow{2}{*}{$\begin{array}{l}\text { Caudal superior parietal } \\
\text { lobule (cSPL) }\end{array}$} & Right & -12 & -74 & 48 & 4.23 & \multirow[t]{2}{*}{0.34} & \multirow[t]{2}{*}{0.15} & $* * *$ & ns. \\
\hline & Left & -18 & -74 & 50 & 4.3 & & & $\mathrm{PES}=0.73, \mathrm{OP}=1.0$ & \\
\hline \multirow[t]{2}{*}{ Ventral premotor cortex (PMv) } & Right & -58 & 2 & 38 & 4.46 & \multirow[t]{2}{*}{0.33} & $* * *$ & $* * *$ & $(\mathrm{H} \times \mathrm{T}) * *$ \\
\hline & Left & -54 & 2 & 32 & 4.91 & & $\mathrm{PES}=0.57, \mathrm{OP}=0.99$ & $\mathrm{PES}=0.70, \mathrm{OP}=1.0$ & $\begin{aligned} \mathrm{PES}= & 0.48, \mathrm{OP}=0.98 \\
& (\mathrm{~T} \times \mathrm{O}) * *\end{aligned}$ \\
\hline \multirow[t]{3}{*}{ Dorsal premotor cortex (PMd) } & Right & -22 & -14 & 64 & 5.26 & $* *$ & $* * *$ & $* * *$ & $\begin{aligned} \mathrm{PES}= & 0.35, \mathrm{OP}=0.86 \\
& (\mathrm{~T} \times \mathrm{O}) *\end{aligned}$ \\
\hline & Left & -26 & -14 & 56 & 4.67 & $\mathrm{PES}=0.41, \mathrm{OP}=0.92$ & $\mathrm{PES}=0.80, \mathrm{OP}=1.0$ & $\mathrm{PES}=0.89, \mathrm{OP}=1.0$ & $\begin{array}{c}\mathrm{PES}=0.26, \mathrm{OP}=0.58 \\
(\mathrm{H} \times \mathrm{T} \times \mathrm{O}) * *\end{array}$ \\
\hline & Right & -36 & 36 & 30 & 2.75 & \multirow{2}{*}{0.49} & $*$ & $* * *$ & $\begin{aligned} \mathrm{PES}= & 0.34, \mathrm{OP}=0.84 \\
& (\mathrm{~T} \times \mathrm{O}) *\end{aligned}$ \\
\hline $\begin{array}{l}\text { Rostral middle frontal gyrus } \\
\text { (rMFG) (right hand from } \\
\text { inclusive contrast) }\end{array}$ & Left & -36 & 36 & 30 & 4.07 & & $\mathrm{PES}=0.21, \mathrm{OP}=0.56$ & $\mathrm{PES}=0.68, \mathrm{OP}=1.0$ & $\mathrm{PES}=0.21, \mathrm{OP}=0.56$ \\
\hline
\end{tabular}

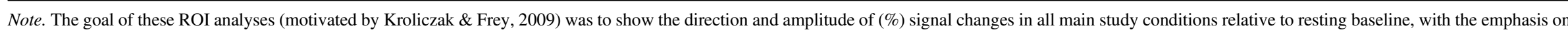

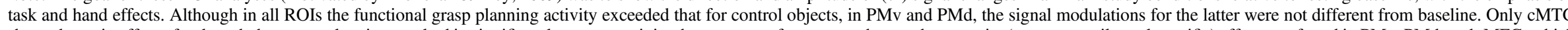

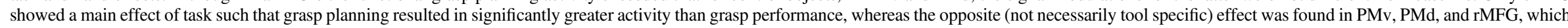

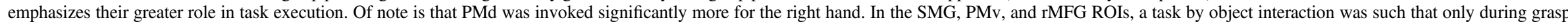

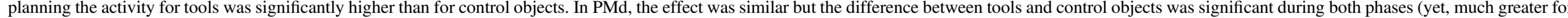

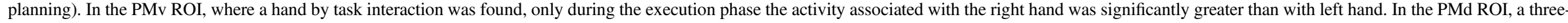

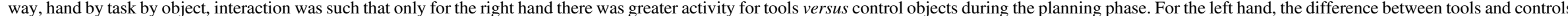

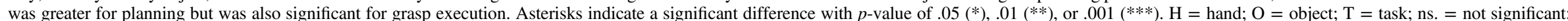
PES = partial eta squared; OP = observed power (computed using $\alpha$ ); the analyses were performed with IBM SPSS Statistics for Macintosh v. 23 (23.0.0.2), Armonk, NY: IBM Corp. 
early visual cortex more than execution of functional grasp (with visual and motor demands controlled). The inverse contrast (not shown) revealed only significant lower-level visual activity and right SPL engagement (likely linked to orientation processing). Finally, as shown in Figure 4C, planning functional grasp compared to its later execution engaged PRN, and right aIPS and SI-MI significantly more. This was the case only when visual and motor demands were controlled. The inverse contrast revealed small clusters of temporal, posterior insular, and visual activity.

\section{Outcomes of ROI Analyses}

The results from ROI analyses are presented in Table 1 and summarized in table caption. With reference to baseline, cMTG and aSMG showed significantly greater involvement in grasp planning, whereas PMv, PMd, and rMFG showed significantly greater activity in grasp execution.

\section{DISCUSSION}

Consistent with earlier reports on pantomimed or real tool use actions (e.g., Brandi et al., 2014; Kroliczak \& Frey, 2009; Valyear et al., 2012), when participants planned functional grasps of tools, greater left-lateralized signal increases were observed in the parieto-frontal regions linked to the PRN. Its involvement was particularly evident when movement kinematics for functional and non-functional grasps were matched. Moreover, in agreement with earlier studies on visual processing of tool features (Bracci, Cavina-Pratesi, Ietswaart, Caramazza, \& Peelen, 2012; Vingerhoets, 2008), when hand/grip kinematics were accounted for, the handindependent temporo-occipital activity associated with visual encoding of tool handles, and the less explicit retrieval of tool concepts and function-relevant features, was almost exclusively left lateralized, too (as in Kroliczak \& Frey, 2009).

The similarities to reports on passive viewing of tools, and actions with real tools, make it unlikely that the effects we observed based on pictorial cues are mainly due to the planning of gestures that only simulate real-life situations. Of course, seeing real tools and planning real grasps directed at them could amplify these outcomes and reveal further regions critical for task performance in real life (Kroliczak, Cavina-Pratesi, Goodman, \& Culham, 2007; Randerath, Goldenberg, Spijkers, Li, \& Hermsdorfer, 2011).

The areas invoked for planning functional grasps were typically located within a network mediating the execution of tool use pantomimes. However, a direct contrast of these tasks demonstrated that grasp planning invoked these regions more. Similarly, with visual and motor processing accounted for, planning functional grasps engaged PRN more than functional grasp execution. Notably, ROI analyses on signals referenced to baseline revealed that as in studies by Kroliczak and collaborators $(2009,2016)$, the temporo-occipital areas (regardless of objects), and SMG (specifically for tools), played much greater roles in the grasp-planning phase.

Conversely, but in line with Vingerhoets and colleagues (2013, 2015), the prefrontal and premotor divisions of PRN contributed substantially more to the execution of the pre-planned grips. Yet, the later effects were not necessarily tool specific and would have emerged in whole brain analyses without common visual and motor processing accounted for. Our results, therefore, indicate that a detection of tool features, the subsequent selection of the required response (Randerath, Li, Goldenberg, \& Hermsdorfer, 2009; Tarhan, Watson, \& Buxbaum, 2015), and the pre-programing of the required movement kinematics (Li, Randerath, Goldenberg, \& Hermsdorfer, 2007; Rosenbaum, Vaughan, Barnes, \& Jorgensen, 1992) may jointly be more demanding than a demonstration of pantomimed functional grasp or even pantomimed tool use, particularly when neural activity not specific to tool processing is taken into account (see also Randerath, Valyear, Hood, \& Frey, 2015).

Although this study shows that PRN is engaged by function-based encoding that precedes the onset of simple grasping (cf. Handjaras et al., 2015), we do not claim this specialized left-lateralized network is not critical for other stages of action performance, including grasping or the actual control of skilled tool use. Indeed, if target objects were constantly seen, grasping tools (pantomimed or real) would most likely involve PRN more than control grasps because some of the computations that are here performed during planning could be completed on-line.

Interestingly, although only more posterior areas of PRN are reported in passive viewing of tools (Macdonald \& Culham, 2015), the areas such as SMA still get involved more for tools, consistent with greater demands for predictive motor planning (Elsinger, Harrington, \& Rao, 2006; Makoshi, Kroliczak, \& van Donkelaar, 2011). All things considered, function-oriented processing seems to be automatically invoked for grasp planning even when no overt tool-use actions are further required, and the underlying neural computations are independent of the hand involved.

\section{Grasping Non-functional versus Functional Objects}

The mechanisms and areas engaged in the control of grasping directed at simple, non-functional objects have been long studied (Binkofski et al., 1998; Culham et al., 2003; Frey, Vinton, Norlund, \& Grafton, 2005; Kroliczak, Westwood, \& Goodale, 2006; Monaco et al., 2010; see also Binkofski \& Buxbaum, 2013; Castiello \& Begliomini, 2008; Gallivan \& Culham, 2015; Goodale, Gonzalez, \& Kroliczak, 2008). The early reports pointed to the critical role of aIPS specialized for the control of hand pre-shaping and/or processing of object shape, and the more caudal divisions of IPS devoted to encoding of object and hand orientation. 
The involvement of the superior parieto-occipital cortex (SPOC) specialized for grasping in peripersonal space has been revealed more recently (Gallivan, Cavina-Pratesi, \& Culham, 2009; Monaco, Sedda, Cavina-Pratesi, \& Culham, 2015). The previous research also compared the effects of using the dominant versus non-dominant hand (Begliomini, Nelini, Caria, Grodd, \& Castiello, 2008) or using hands versus a simple "gripper" for grasping non-functional objects (Jacobs, Danielmeier, \& Frey, 2010). Yet, none of these studies, including the latter report, successfully revealed greater involvement of PRN, particularly cMTG, aSMG, and rMFG in the examined tasks. The only regions quite consistently reported were aIPS, PMd, and/or SPOC.

Our study shows that, independent of the to-be-used hand, the initial phases of tool processing for functional grasp planning engage PRN more than non-tools, with conceptual/ functional processing and response selection managed, respectively, by the temporo-occipital and inferior parietal regions. Notably, although the differences between the control of tool and non-tool grasps dissipate during grip execution, most likely because the grasp points are selected and thinking about a tool as such is no longer needed, undoubtedly further local computations within PRN would be required both for actual grasping and tool use actions. Yet, the PRN, particularly the left posterior parietal cortex, would then be invoked for different reasons, such as the sequencing and fine-tuning of movement progression for adequate functional grasp, and later tool use and/or feedback on the effective guidance of such movements, including their timing (Harrington \& Haaland, 1991; Harrington et al., 2000; Kimura \& Archibald, 1974; Rao et al., 1997; cf. Ritterband-Rosenbaum, Hermosillo, Kroliczak, \& van Donkelaar, 2014).

Because in the critical comparisons the characteristics of graspable parts and the required movement kinematics for tools and non-tools were matched, task difficulty such as a need for more precise and deliberate grasp of a tool cannot solely account for the observed engagement of PRN. However, closer visual inspection might be required for tools. As in a study by Macdonald and Culham (2015), the overall object appearance should not play a critical role, either, because the vast majority of stimuli were carefully tallied for size, length, and the number of parts.

It is also unlikely that an automatic silent naming of tools would invoke PRN more because the control objects could be also named (e.g., a hammer shaft or twig), although the names would often come from a different level of categorization (Chao, Weisberg, \& Martin, 2002). Thus, even though the grip task quickly diverted participants' attention from the functional parts of tools, the spontaneous, often implicit affordance processing could be one of the major factors here. After all, attentional resources and the closely related first saccades are instinctively directed toward functional, rather than graspable, parts of tools (Belardinelli, Barabas, Himmelbach, \& Butz, 2016; Kourtis \& Vingerhoets, 2015). In sum, the neural computations within PRN, including attentional resources, would be automatically tuned to object functionality which is critical for initiating a cascade of further prerequisites necessary for effective planning of functional grasps.

\section{Affordances and Their Role in Hand-Object Interactions}

From the time of Gibson, functionality of objects is associated with the aforementioned concept of affordances (Gibson, 1977, 1986) typically referring to such characteristics of tools that automatically invoke the most effective actions in their presence. Regardless of whether the general macro-affordances (Mizelle et al., 2013) or more specific micro-affordances (Pellicano, Iani, Borghi, Rubichi, \& Nicoletti, 2010; Michalowski \& Kroliczak, 2015) are considered, with a notable exception of behavioral studies by Ellis and Tucker (2000), the focus has been usually put on processing of the goal/target-relevant parts of tools, and their impact on other objects.

Our results indicate that PRN's contribution to the control of functional grasp depends critically on such early affordance-based visual processing and knowledge on their functions. These outcomes do not resolve an issue of neuropsychological evidence for dissociable representations for grasping versus using tools (Randerath et al., 2010). Nonetheless, we have evidence that the former skill may depend more on the integrity of the parieto-frontal pathways of PRN, whereas the latter is also modulated by inputs from the precuneus, anterior temporal, and dorsal lateral/medial prefrontal cortices.

\section{CONCLUSIONS}

This study shows that even a simple task of planning functional grasp invokes the PRN typically associated with tool use skills. This is not surprising because quite sophisticated cognitive mechanisms are required for affordance-based visual processing. Undoubtedly, planning grasps of tools involves weighting of several factors, with priority given to processing of structural features of graspable parts, and their transient positions that affect biomechanical costs of grip performances. Yet, all this happens in the context of the automatic retrieval of our knowledge about tools, and experience with handling and using them. Such a simple task but reveals the core of motor cognition.

\section{ACKNOWLEDGMENTS}

The authors declare no conflict of interest. This work was supported by National Science Centre (Narodowe Centrum Nauki, NCN) grant Maestro 2011/02/A/HS6/00174 to Gregory Króliczak. The equipment used for data acquisition and analyses was funded by the Ministry of Science and Higher Education (Ministerstwo Nauki i Szkolnictwa Wyższego, MNiSW) grant 6168/IA/128/2012 to 
Gregory Króliczak. During all stages of this work $Ł P$ and GK were also supported by the Maestro grant. The CePT research infrastructure was purchased with funds from the European Regional Development Fund as part of the Innovative Economy Operational Programme, 2007-2013. We thank Dr. Mikołaj Pawlak for assistance with setting scanning sequences/parameters, and help with preliminary data acquisition. We would also like to thank Szymon $P$. Biduła for setting the data preprocessing pipeline, and help with the analyses of the localizer scans. In recognition of their work, Mikołaj Pawlak and Szymon P. Biduła were also supported by the Maestro grant. Finally, the authors would like to acknowledge Sebastian Seyda for help with subject recruitment. Author contributions: This project was conceptualized by G.K. and Ł.P. Data were collected by Ł.P. and G.K., analyzed by Ł.P. and G.K., and interpreted by both authors. The manuscript was written by G.K. and Ł.P.

\section{Supplementary material}

To view supplementary material for this article, please visit https://doi.org/10.1017/S1355617716001120

\section{REFERENCES}

Beckmann, C.F., Jenkinson, M., \& Smith, S.M. (2003). General multilevel linear modeling for group analysis in FMRI. Neuroimage, 20(2), 1052-1063. doi: 10.1016/S1053-8119(03) 00435-X

Begliomini, C., Nelini, C., Caria, A., Grodd, W., \& Castiello, U. (2008). Cortical activations in humans grasp-related areas depend on hand used and handedness. PLoS One, 3(10), e3388.

Belardinelli, A., Barabas, M., Himmelbach, M., \& Butz, M.V. (2016). Anticipatory eye fixations reveal tool knowledge for tool interaction. Experimental Brain Reseach, 234, 2415-2431. doi: 10.1007/s00221-016-4646-0

Bidula, S.P., \& Kroliczak, G. (2015). Structural asymmetry of the insula is linked to the lateralization of gesture and language. European Journal of Neuroscience, 41(11), 1438-1447. doi: 10.1111/ejn.12888

Binkofski, F., \& Buxbaum, L.J. (2013). Two action systems in the human brain. Brain and Language, 127(2), 222-229. doi: 10.1016/j.band1.2012.07.007

Binkofski, F., Dohle, C., Posse, S., Stephan, K.M., Hefter, H., Seitz, R.J., \& Freund, H.J. (1998). Human anterior intraparietal area subserves prehension: A combined lesion and functional MRI activation study. Neurology, 50(5), 1253-1259. doi: 10.1212/WNL.50.5.1253

Bracci, S., Cavina-Pratesi, C., Ietswaart, M., Caramazza, A., \& Peelen, M.V. (2012). Closely overlapping responses to tools and hands in left lateral occipitotemporal cortex. Journal of Neurophysiology, 107(5), 1443-1456. doi: 10.1152/jn.00 619.2011

Brandi, M.L., Wohlschlager, A., Sorg, C., \& Hermsdorfer, J. (2014). The neural correlates of planning and executing actual tool use. The Journal of Neuroscience, 34(39), 13183-13194. doi: 10.1523/JNEUROSCI.0597-14.2014

Buxbaum, L.J., Kyle, K.M., Tang, K., \& Detre, J.A. (2006). Neural substrates of knowledge of hand postures for object grasping and functional object use: Evidence from fMRI. Brain Research, 1117(1), 175-185. doi: 10.1016/j.brainres.2006.08.010
Buxbaum, L.J., Shapiro, A.D., \& Coslett, H.B. (2014). Critical brain regions for tool-related and imitative actions: A componential analysis. Brain, 137(Pt 7), 1971-1985. doi: 10.1093/brain/ awu111

Castiello, U., \& Begliomini, C. (2008). The cortical control of visually guided grasping. Neuroscientist, 14(2), 157-170.

Chao, L.L., Weisberg, J., \& Martin, A. (2002). Experiencedependent modulation of category-related cortical activity. Cereb Cortex, 12(5), 545-551.

Creem-Regehr, S.H., \& Lee, J.N. (2005). Neural representations of graspable objects: Are tools special? Brain Research. Cognitive Brain Research, 22(3), 457-469.

Culham, J.C., Danckert, S.L., DeSouza, J.F., Gati, J.S., Menon, R. S., \& Goodale, M.A. (2003). Visually guided grasping produces fMRI activation in dorsal but not ventral stream brain areas. Experimental Brain Research, 153(2), 180-189. doi: 10.1007/ s00221-003-1591-5

Eklund, A., Nichols, T.E., \& Knutsson, H. (2016). Cluster failure: Why fMRI inferences for spatial extent have inflated falsepositive rates. Proceedings of the National Academy of Sciences of the United States of America, 113(28), 7900-7905. doi: $10.1073 /$ pnas.1602413113

Ellis, R., \& Tucker, M. (2000). Micro-affordance: The potentiation of components of action by seen objects. British Journal of Psychology, 91(Pt 4), 451-471.

Elsinger, C.L., Harrington, D.L., \& Rao, S.M. (2006). From preparation to online control: Reappraisal of neural circuitry mediating internally generated and externally guided actions. Neuroimage, 31(3), 1177-1187.

Fabbri, S., Stubbs, K.M., Cusack, R., \& Culham, J.C. (2016). Disentangling Representations of Object and Grasp Properties in the Human Brain. The Journal of Neuroscience, 36(29), 7648-7662. doi: 10.1523/JNEUROSCI.0313-16.2016

Fischl, B. (2012). FreeSurfer. Neuroimage, 62(2), 774-781. doi: 10.1016/j.neuroimage.2012.01.021

Frey, S.H. (2007). What puts the how in where? Tool use and the divided visual streams hypothesis. Cortex, 43(3), 368-375.

Frey, S.H. (2008). Tool use, communicative gesture and cerebral asymmetries in the modern human brain. Philosophical Transactions of the Royal Society of London. Series B, Biological Sciences, 363(1499), 1951-1957. doi: 10.1098/ rstb.2008.0008

Frey, S.H., Vinton, D., Norlund, R., \& Grafton, S.T. (2005). Cortical topography of human anterior intraparietal cortex active during visually guided grasping. Brain Research. Cognitive Brain Research, 23(2-3), 397-405. doi: 10.1016/j.cogbrainres.2004.11.010

Gallivan, J.P., Cavina-Pratesi, C., \& Culham, J.C. (2009). Is that within reach? fMRI reveals that the human superior parietooccipital cortex encodes objects reachable by the hand. Journal of Neuroscience, 29(14), 4381-4391. doi: 10.1523/JNEUR OSCI.0377-09.2009

Gallivan, J.P., \& Culham, J.C. (2015). Neural coding within human brain areas involved in actions. Current Opinion in Neurobiology, 33, 141-149. doi: 10.1016/j.conb.2015.03.012

Garofeanu, C., Kroliczak, G., Goodale, M.A., \& Humphrey, G.K. (2004). Naming and grasping common objects: A priming study. Experimental Brain Research, 159(1), 55-64. doi: 10.1007/ s00221-004-1932-z

Gibson, J.J. (1977). The theory of affordances. In R. Shaw \& J. Bransford (Eds.), Perceiving, acting, and knowing. Toward an ecological psychology (pp. 67-82). Hillsdale, NJ: Lawrence Erlbaum Associates. 
Gibson, J.J. (1986). The ecological approach to visual perception. Hillsdale, NJ: Lawrence Erlbaum Associates.

Goldenberg, G., Hartmann, K., \& Schlott, I. (2003). Defective pantomime of object use in left brain damage: Apraxia or asymbolia? Neuropsychologia, 41(12), 1565-1573.

Goldenberg, G., \& Spatt, J. (2009). The neural basis of tool use. Brain, 132(Pt 6), 1645-1655. doi: 10.1093/brain/awp080

Goodale, M.A., Gonzalez, C.L., \& Kroliczak, G. (2008). Action rules: Why the visual control of reaching and grasping is not always influenced by perceptual illusions. Perception, 37(3), 355-366. doi: 10.1068/p5876

Goodale, M.A., Kroliczak, G., \& Westwood, D.A. (2005). Dual routes to action: Contributions of the dorsal and ventral streams to adaptive behavior. Progress in Brain Research, 149, 269-283. doi: 10.1016/S0079-6123(05)49019-6

Haaland, K.Y., \& Harrington, D.L. (1996). Hemispheric asymmetry of movement. Current Opinion in Neurobiology, 6(6), 796-800.

Haaland, K.Y., Harrington, D.L., \& Knight, R.T. (2000). Neural representations of skilled movement. Brain, 123, 2306-2313. doi: 10.1093/brain/123.11.2306

Handjaras, G., Bernardi, G., Benuzzi, F., Nichelli, P.F., Pietrini, P., \& Ricciardi, E. (2015). A topographical organization for action representation in the human brain. Human Brain Mapping, 36(10), 3832-3844. doi: 10.1002/hbm.22881

Harrington, D.L., \& Haaland, K.Y. (1991). Hemispheric specialization for motor sequencing: Abnormalities in levels of programming. Neuropsychologia, 29(2), 147-163.

Harrington, D.L., Rao, S.M., Haaland, K.Y., Bobholz, J.A., Mayer, A.R., Binderx, J.R., \& Cox, R.W. (2000). Specialized neural systems underlying representations of sequential movements. Journal of Cognitive Neuroscience, 12(1), 56-77.

Hermsdorfer, J., Terlinden, G., Muhlau, M., Goldenberg, G., \& Wohlschlager, A.M. (2007). Neural representations of pantomimed and actual tool use: Evidence from an event-related fMRI study. Neuroimage, 36(Suppl 2), T109-T118. doi: 10.1016/j.neuroimage.2007.03.037

Ishibashi, R., Pobric, G., Saito, S., \& Lambon Ralph, M.A. (2016). The neural network for tool-related cognition: An activation likelihood estimation meta-analysis of 70 neuroimaging contrasts. Cognitive Neuropsychology, 33(3-4), 241-256. doi: 10.1080/02643294.2016.1188798

Jacobs, S., Danielmeier, C., \& Frey, S.H. (2010). Human anterior intraparietal and ventral premotor cortices support representations of grasping with the hand or a novel tool. Journal of Cognitive Neuroscience, 22(11), 2594-2608. doi: 10.1162/jocn.2009.21372

Jenkinson, M., Beckmann, C.F., Behrens, T.E., Woolrich, M.W., \& Smith, S.M. (2012). FSL. Neuroimage, 62(2), 782-790. doi: 10.1016/j.neuroimage.2011.09.015

Johnson-Frey, S.H., Newman-Norlund, R., \& Grafton, S.T. (2005). A distributed left hemisphere network active during planning of everyday tool use skills. Cerebral Cortex, 15(6), 681-695. doi: 10.1093/cercor/bhh169

Kimura, D., \& Archibald, Y. (1974). Motor functions of the left hemisphere. Brain, 97(2), 337-350. doi: 10.1093/brain/97.1.337

Kourtis, D., \& Vingerhoets, G. (2015). Perceiving objects by their function: An EEG study on feature saliency and prehensile affordances. Biological Psychology, 110, 138-147.

Kristensen, S., Garcea, F.E., Mahon, B.Z., \& Almeida, J. (2016). Temporal Frequency Tuning Reveals Interactions between the Dorsal and Ventral Visual Streams. Journal of Cognitive Neuroscience, 28(9), 1295-1302. doi: 10.1162/jocn_a_00969
Kroliczak, G., Cavina-Pratesi, C., Goodman, D.A., \& Culham, J.C. (2007). What does the brain do when you fake it? An FMRI study of pantomimed and real grasping. Journal of Neurophysiology, 97(3), 2410-2422. doi: 10.1152/jn.00778.2006

Kroliczak, G., \& Frey, S.H. (2009). A common network in the left cerebral hemisphere represents planning of tool use pantomimes and familiar intransitive gestures at the handindependent level. Cerebral Cortex, 19(10), 2396-2410. doi: 10.1093/cercor/bhn261

Kroliczak, G., McAdam, T.D., Quinlan, D.J., \& Culham, J.C. (2008). The human dorsal stream adapts to real actions and 3D shape processing: A functional magnetic resonance imaging study. Journal of Neurophysiology, 100(5), 2627-2639. doi: 10.1152/jn.01376.2007

Kroliczak, G., Piper, B.J., \& Frey, S.H. (2016). Specialization of the left supramarginal gyrus for hand-independent praxis representation is not related to hand dominance. Neuropsychologia. doi: 10.1016/j.neuropsychologia.2016.03.023

Kroliczak, G., Westwood, D.A., \& Goodale, M.A. (2006). Differential effects of advance semantic cues on grasping, naming, and manual estimation. Experimental Brain Research, 175(1), 139-152. doi: 10.1007/s00221-006-0524-5

Kubiak, A., \& Kroliczak, G. (2016). Left extrastriate body area is sensitive to the meaning of symbolic gesture: Evidence from fMRI repetition suppression. Scientific Reports, 6, 31064. doi: 10.1038/srep31064

Li, Y., Randerath, J., Goldenberg, G., \& Hermsdorfer, J. (2007). Grip forces isolated from knowledge about object properties following a left parietal lesion. Neuroscience Letters, 426(3), 187-191. doi: 10.1016/j.neulet.2007.09.008

Macdonald, S.N., \& Culham, J.C. (2015). Do human brain areas involved in visuomotor actions show a preference for real tools over visually similar non-tools? Neuropsychologia, 77, 35-41. doi: 10.1016/j.neuropsychologia.2015.08.004

Maki-Marttunen, V., Villarreal, M., \& Leiguarda, R.C. (2014). Lateralization of brain activity during motor planning of proximal and distal gestures. Behavioural Brain Research, 272, 226-237. doi: 10.1016/j.bbr.2014.06.055

Makoshi, Z., Kroliczak, G., \& van Donkelaar, P. (2011). Human supplementary motor area contribution to predictive motor planning. Journal of Motor Behavior, 43(4), 303-309. doi: 10.1080/00222895.2011.584085

Marangon, M., Kubiak, A., \& Kroliczak, G. (2016). Haptically guided grasping. fMRI shows right-hemisphere parietal stimulus encoding, and bilateral dorso-ventral parietal gradients of object- and action-related processing during grasp execution. Frontiers in Human Neuroscience, 9, 691. doi: 10.3389/ fnhum.2015.00691

Michalowski, B., \& Kroliczak, G. (2015). Sinistrals are rarely "right": Evidence from tool-affordance processing in visual half-field paradigms. Frontiers in Human Neuroscience, 9, 166. doi: 10.3389/fnhum.2015.00166

Miezin, F.M., Maccotta, L., Ollinger, J.M., Petersen, S.E., \& Buckner, R.L. (2000). Characterizing the hemodynamic response: Effects of presentation rate, sampling procedure, and the possibility of ordering brain activity based on relative timing. Neuroimage, 11(6 Pt 1), 735-759. doi: 10.1006/nimg. 2000.0568

Mizelle, J.C., Kelly, R.L., \& Wheaton, L.A. (2013). Ventral encoding of functional affordances: A neural pathway for identifying errors in action. Brain and Cognition, 82(3), 274-282. doi: 10.1016/j.bandc.2013.05.002 
Monaco, S., Kroliczak, G., Quinlan, D.J., Fattori, P., Galletti, C., Goodale, M.A., \& Culham, J.C. (2010). Contribution of visual and proprioceptive information to the precision of reaching movements. Experimental Brain Research, 202(1), 15-32. doi: 10.1007/s00221-009-2106-9

Monaco, S., Sedda, A., Cavina-Pratesi, C., \& Culham, J.C. (2015). Neural correlates of object size and object location during grasping actions. European Journal of Neuroscience, 41(4), 454-465. doi: 10.1111/ejn.12786

Nichols, T., Brett, M., Andersson, J., Wager, T., \& Poline, J.B. (2005). Valid conjunction inference with the minimum statistic. Neuroimage, 25(3), 653-660. doi: 10.1016/j.neuroimage.2004. 12.005

Oldfield, R.C. (1971). The assessment and analysis of handedness: The Edinburgh inventory. Neuropsychologia, 9, 97-113. doi: 10.1016/0028-3932(71)90067-4

Peeters, R.R., Rizzolatti, G., \& Orban, G.A. (2013). Functional properties of the left parietal tool use region. Neuroimage, 78, 83-93. doi: 10.1016/j.neuroimage.2013.04.023

Pellicano, A., Iani, C., Borghi, A.M., Rubichi, S., \& Nicoletti, R. (2010). Simon-like and functional affordance effects with tools: The effects of object perceptual discrimination and object action state. Quarterly Journal of Experimental Psychology (Hove), 63(11), 2190-2201. doi: 10.1080/17470218.2010.486903

Raichle, M.E., MacLeod, A.M., Snyder, A.Z., Powers, W.J., Gusnard, D.A., \& Shulman, G.L. (2001). A default mode of brain function. Proceedings of the National Academy of Sciences of the United States of America, 98(2), 676-682.

Randerath, J., Goldenberg, G., Spijkers, W., Li, Y., \& Hermsdorfer, J. (2010). Different left brain regions are essential for grasping a tool compared with its subsequent use. Neuroimage, 53(1), 171-180. doi: 10.1016/j.neuroimage.2010.06.038

Randerath, J., Goldenberg, G., Spijkers, W., Li, Y., \& Hermsdorfer, J. (2011). From pantomime to actual use: How affordances can facilitate actual tool-use. Neuropsychologia, 49(9), 2410-2416. doi: 10.1016/j.neuropsychologia.2011.04.017

Randerath, J., Li, Y., Goldenberg, G., \& Hermsdorfer, J. (2009). Grasping tools: Effects of task and apraxia. Neuropsychologia, 47(2), 497-505. doi: 10.1016/j.neuropsychologia.2008.10.005

Randerath, J., Valyear, K.F., Hood, A., \& Frey, S.H. (2015). Two routes to the same action: An action repetition priming study. Journal of Motor Behavior, 47(2), 142-152. doi: 10.1080/ 00222895.2014.961891

Rao, S.M., Harrington, D.L., Haaland, K.Y., Bobholz, J.A., Cox, R. W., \& Binder, J.R. (1997). Distributed neural systems underlying the timing of movements. Journal of Neuroscience, 17(14), 5528-5535.

Ritterband-Rosenbaum, A., Hermosillo, R., Kroliczak, G., \& van Donkelaar, P. (2014). Hand position-dependent modulation of errors in vibrotactile temporal order judgments: The effects of transcranial magnetic stimulation to the human posterior parietal cortex. Experimental Brain Research, 232(6), 1689-1698. doi: 10.1007/s00221-014-3861-9
Rosenbaum, D.A., Vaughan, J., Barnes, H.J., \& Jorgensen, M.J. (1992). Time course of movement planning: Selection of handgrips for object manipulation. Journal of Experimental Psychology: Learning, Memory and Cognition, 18(5), 1058-1073.

Tarhan, L.Y., Watson, C.E., \& Buxbaum, L.J. (2015). Shared and distinct neuroanatomic regions critical for tool-related action production and recognition: evidence from 131 left-hemisphere stroke patients. Journal of Cognitive Neuroscience, 27(12), 2491-2511. doi: 10.1162/jocn_a_00876

Valyear, K.F., Gallivan, J.P., McLean, D.A., \& Culham, J.C. (2012). fMRI repetition suppression for familiar but not arbitrary actions with tools. Journal of Neuroscience, 32(12), 4247-4259. doi: 10.1523/JNEUROSCI.5270-11.2012

Van Essen, D.C. (2005). A Population-Average, Landmark- and Surface-based (PALS) atlas of human cerebral cortex. Neuroimage, 28(3), 635-662. doi: 10.1016/j.neuroimage.2005.06.058

Vannuscorps, G., Dricot, L., \& Pillon, A. (2016). Persistent sparing of action conceptual processing in spite of increasing disorders of action production: A case against motor embodiment of action concepts. Cognitive Neuropsychology, 33, 191-219. doi: 10.1080/02643294.2016.1186615

Vingerhoets, G. (2008). Knowing about tools: Neural correlates of tool familiarity and experience. Neuroimage, 40(3), 1380-1391. doi: 10.1016/j.neuroimage.2007.12.058

Vingerhoets, G. (2014). Contribution of the posterior parietal cortex in reaching, grasping, and using objects and tools. Frontiers in Psychology, 5, 151. doi: 10.3389/fpsyg.2014.00151

Vingerhoets, G., Acke, F., Alderweireldt, A.S., Nys, J., Vandemaele, P., \& Achten, E. (2012). Cerebral lateralization of praxis in right- and left-handedness: Same pattern, different strength. Human Brain Mapping, 33(4), 763-777. doi: 10.1002/ hbm. 21247

Vingerhoets, G., \& Clauwaert, A. (2015). Functional connectivity associated with hand shape generation: Imitating novel hand postures and pantomiming tool grips challenge different nodes of a shared neural network. Human Brain Mapping, 36(9), 3426-3440. doi: 10.1002/hbm.22853

Vingerhoets, G., Nys, J., Honore, P., Vandekerckhove, E., \& Vandemaele, P. (2013). Human left ventral premotor cortex mediates matching of hand posture to object use. PLoS One, 8(7), e70480. doi: 10.1371/journal.pone.0070480

Vingerhoets, G., Vandekerckhove, E., Honore, P., Vandemaele, P., \& Achten, E. (2011). Neural correlates of pantomiming familiar and unfamiliar tools: Action semantics versus mechanical problem solving? Human Brain Mapping, 32(6), 905-918. doi: $10.1002 / \mathrm{hbm} .21078$

Watson, C.E., \& Buxbaum, L.J. (2015). A distributed network critical for selecting among tool-directed actions. Cortex, 65, 65-82. doi: 10.1016/j.cortex.2015.01.007

Woolrich, M.W., Ripley, B.D., Brady, M., \& Smith, S.M. (2001). Temporal autocorrelation in univariate linear modeling of FMRI data. Neuroimage, 14(6), 1370-1386. doi: 10.1006/ nimg.2001.0931 